Review

\title{
Language activation distributions revealed by fMRI in post-operative epilepsy patients: Differences between left- and right-sided resections
}

\author{
W.H. Backes ${ }^{\mathrm{a}, *}$, K. Deblaere ${ }^{\mathrm{g}}$, K. Vonck ${ }^{\mathrm{g}}$, A.G. Kessels ${ }^{\mathrm{b}}$, P. Boon ${ }^{\mathrm{c}}$, \\ P. Hofman ${ }^{\text {a }}$, J.T. Wilmink ${ }^{\text {a }}$, G. Vingerhoets ${ }^{\text {h }}$, P.A. Boon ${ }^{\text {g,f }}$, \\ R. Achten ${ }^{\mathrm{g}}$, J. Vermeulen ${ }^{\mathrm{e}}$, A.P. Aldenkamp ${ }^{\mathrm{d}, \mathrm{f}}$ \\ a Department of Radiology, Maastricht University Hospital, P. Debyelaan 25, NL-6229 HX Maastricht, The Netherlands \\ ${ }^{\mathrm{b}}$ Department of Epidemiology and Medical Technological Assessment, Maastricht University Hospital, \\ P. Debyelaan 25, NL-6229 HX Maastricht, The Netherlands \\ ${ }^{\mathrm{c}}$ Department of Clinical Psychology, Maastricht University Hospital, P. Debyelaan 25, NL-6229 HX Maastricht, The Netherlands \\ ${ }^{\mathrm{d}}$ Department of Neurology, Maastricht University Hospital, P. Debyelaan 25, NL-6229 HX Maastricht, The Netherlands \\ e S.E.I.N., Heemstede, The Netherlands \\ ${ }^{\mathrm{f}}$ Epilepsy Center Kempenhaeghe, Heeze, The Netherlands \\ ${ }^{\mathrm{g}}$ Department of Radiology, Ghent University Hospital and Ghent University, Ghent, Belgium \\ ${ }^{\mathrm{h}}$ Department of Neuropsychology, Ghent University Hospital and Ghent University, Ghent, Belgium
}

Received 1 June 2005; received in revised form 12 June 2005; accepted 22 June 2005

Available online 22 August 2005

\begin{abstract}
Objective: To reveal differences of cerebral activation related to language functions in post-operative temporal lobe epilepsy (TLE) patients.

Methods: Right (RTL) and left temporal lobe (LTL) resected patients, and healthy controls were studied using functional magnetic resonance imaging (fMRI). Only patients with complete left-hemispheric language dominance according to the intracarotid amytal procedure (IAP) were included. Language-related activations were evoked by performing word generation and text reading language tasks. Activation lateralization and temporo-frontal distribution effects were analysed.

Results: For word generation, only LTL patients showed reduced left lateralized activation compared to controls, due to a decrease in activation in the left prefrontal cortex and an increase in the right prefrontal cortex. For reading, the left-hemispheric lateralization in RTL patients increased because of enhanced activity in the left prefrontal cortex, whereas for LTL patients the activation became bilaterally distributed over the temporal lobes. Lateralization results between pre-operative IAP and postoperative fMRI were highly discordant. Significant temporo-frontal distribution changes manifested from the reading but not from the word generation task.
\end{abstract}

\footnotetext{
* Corresponding author. Tel.: +31 43 3876948; fax: +31 433876909 .

E-mail address:wbac@rdia.azm.nl (W.H. Backes).
} 
Conclusion: The cerebral language representation in post-operative LTL epilepsy patients is more bi-hemispherically lateralized than in controls and RTL patients. Post-operative temporo-frontal and interhemispheric redistribution effects, involving contralateral homologuous brain areas, are suggested to contribute to the cerebral reorganisation of language function.

(C) 2005 Elsevier B.V. All rights reserved.

Keywords: Epilepsy; Anterior temporal lobectomy; fMRI; Language

\section{Contents}

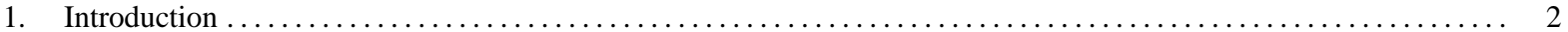

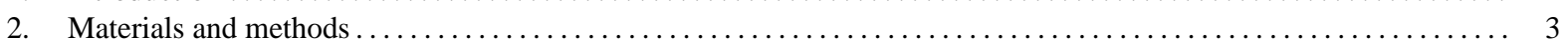

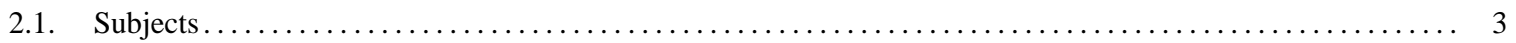

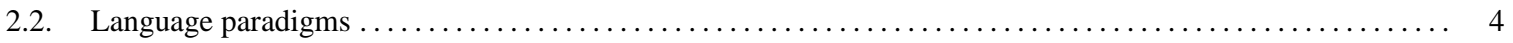

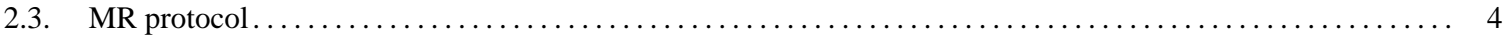

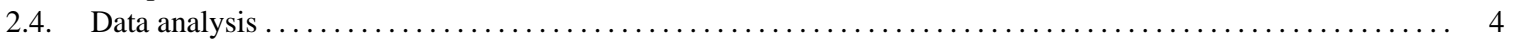

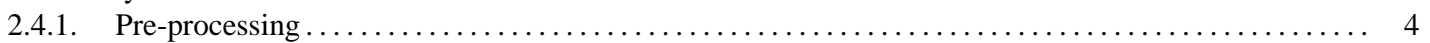

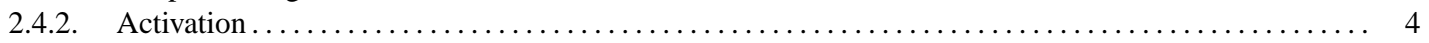

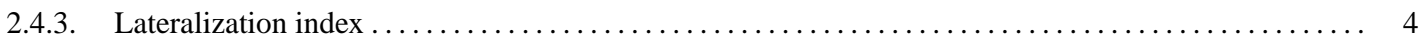

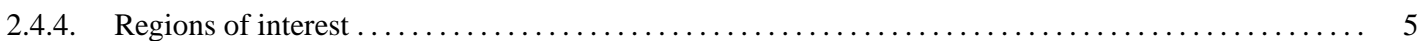

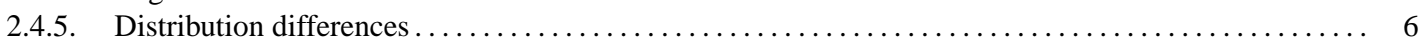

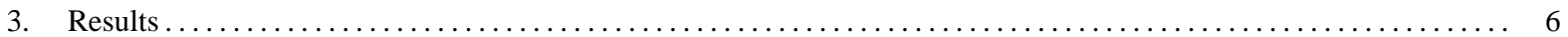

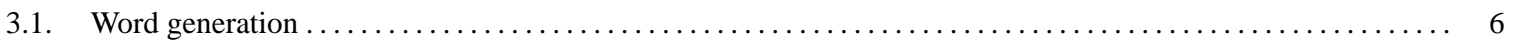

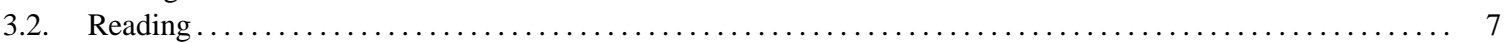

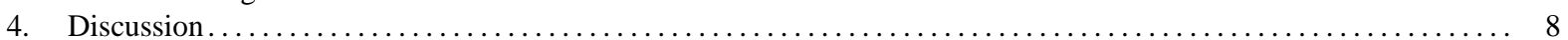

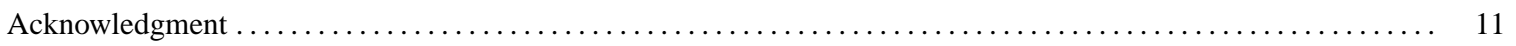

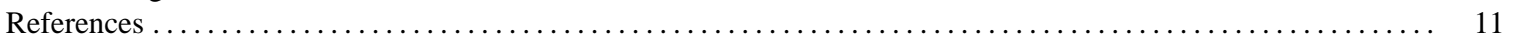

\section{Introduction}

Anterior temporal lobectomy (ATL) is a wellestablished surgical treatment for pharmaco-resistant temporal lobe epilepsy. The surgical procedure results in either a significant reduction in seizure frequency or complete seizure remission in about $70 \%$ of the patients. The resection involves parts of the unilateral mesial temporal cortex as well as lateral neocortical structures of the anterior temporal lobe. The resection may disturb or damage cortex eloquent for memory and language functioning. Despite extensive non-invasive and invasive pre-operative hemispheric language and memory testing, subtle problems may arise in post-operative language functioning after left ATL (Hermann et al., 1994; Davies et al., 1998; Langfitt and Rausch, 1996; Clusmann et al., 2002). More frequently difficulties in verbal memory are found post-operatively (Rausch et al., 2003; Lee et al., 2002; Bell and Davies, 1998). When a hippocampal sclerosis is diagnosed less post-operative difficulties are found, presumably caused by reorganization of function during the transformation of healthy brain tissue into a sclerosis. Consequently, affected neural circuits may already pre-operatively reorganize to distant brain regions.

Typically, the left hemisphere is dominant for language function, while complete right-hemispheric dominance is rare (about 1-2\%) (8). Bilateral language representation is found far less (about 20\%) than left-hemispheric dominance (Loring et al., 1990). Remarkably, bilateral language representation is found to be more prevalent in patients with epilepsy than in healthy people. This, again, is assumed to be caused by neuronal reorganization as a consequence of early left hemisphere epilepsies and lesions. Evidence exists that 
functional reorganization enables the right hemisphere to mediate language functions in response to left brain pathology early in life (Helmstaedter et al., 1997).

Functional MRI is an imaging technique that provides unique and highly reproducible (Rutten et al., 2002a,b) means to localize the brain systems involved in processing language functions. For diagnostic language and memory evaluation in patients with epilepsy, a number of research centres (Baxendale, 2002) has recently correlated the outcomes of fMRI and the IAP (i.e. the Wada test), which is considered the gold standard for language lateralization. Until now, extremely good correlations have been published between fMRI and the IAP, especially for epilepsy patients with a typical (i.e. left dominant) language representation (Binder et al., 1996; Yetkin et al., 1998; Lehéricy et al., 2000; Rutten et al., 2002a,b; Spreer et al., 2002; Adcock et al., 2003; Woermann et al., 2003).

The number of imaging studies dealing with postlesional plasticity of the brain is, however, small and limited to case studies. Hertz-Pannier et al. (2002) recently reported in a serial fMRI study an interhemispheric transfer of brain activation in response to language demands in a 9-year-old child after left hemispherotomy. The authors explained the plasticity effect by the assumption of a pre-existing bilateral language network that is inhibited by preferential left activation in the normal healthy situation. Shifts in brain activation patterns are also found in TLE patients with lesions at or near the language areas (Adcock et al., 2003; Springer et al., 1999).

This study aimed at detecting reorganizations in language activation patterns in a group of patients with epilepsy after ATL. The hypothesis is that when no cerebral language reorganizations occur the activation patterns should be independent of the resection side. To test this an fMRI study was set out that compared the brain activation patterns between patient groups who had a left- and right-sided resection against controls. By using word generation and reading as neuropsychological language tasks brain systems were studied that respond to expressive and receptive language functioning, respectively.

\section{Materials and methods}

\subsection{Subjects}

Characteristics of the patients and the healthy controls are summarized in Table 1. Nine healthy controls, 12 RTL and 9 LTL resected epilepsy patients were included in the study protocol. All patients and controls were right-handed. The patients underwent a presurgical language and memory evaluation with the IAP (Wada and Rasmussen, 1960). During the IAP amobarbital, a short acting anaesthetic, is injected in one of the internal carotid arteries producing a transient anaesthesia of the territories of the middle and anterior cerebral arteries of the ipsilateral hemisphere. Several tests are then performed to evaluate language lateralization. The language part of this test comprised overt object naming, picture description, and story recall. Only patients with complete left-hemispheric language dominance were included in this study. All patients underwent a partial resection of the anterior temporal lobe with

Table 1

Characteristics of epilepsy patients and controls

\begin{tabular}{|c|c|c|c|}
\hline Characteristic & Controls & RTL & LTL \\
\hline Male/female & $3 / 9$ & $5 / 7$ & $3 / 6$ \\
\hline Age at onset epilepsy (year) & & $12.4 \pm 7.6,12(4-31)$ & $10.7 \pm 6.8,8.5(3-22)$ \\
\hline Age at surgery (year) & & $30 \pm 8,31(18-43)$ & $34 \pm 9,33(23-48)$ \\
\hline Age at imaging (year) & $26 \pm 6$ & $34 \pm 7,35(23-46)$ & $37 \pm 8,36.5(26-52)$ \\
\hline Time between surgery and fMRI (year) & & $4.5 \pm 2.0,5(1-8)$ & $3.8 \pm 3.4,3.5(1-11)$ \\
\hline Seizure frequency $\left(\right.$ month $^{-1}$ ) & & $3.5 \pm 1.2,4(1-4)$ & $4.2 \pm 2.4,4(1-4)$ \\
\hline Presurgical PIQ & & $112 \pm 15,116(88-134)$ & $109 \pm 13,110(82-126)$ \\
\hline Presurgical VIQ & & $105 \pm 15,108(82-133)$ & $103 \pm 17,111(71-122)$ \\
\hline Resection volume $\left(\mathrm{cm}^{3}\right)$ & & $25.5 \pm 10.1,27(8.9-42)$ & $16.9 \pm 5.5,17(9.9-24)$ \\
\hline Pathology & & $7 \mathrm{HS}, 1 \mathrm{HT}, 4 \mathrm{OP}$ & $7 \mathrm{HS}, 1 \mathrm{HT}, 1 \mathrm{OP}$ \\
\hline
\end{tabular}

Values are mean \pm 1 S.D., median (range). LTL: left temporal lobe; RTL: right temporal lobe; PIQ: performance intelligence quotient; VIQ: verbal intelligence quotient; HS: hippocampal sclerosis; HT hippocampal tumor; OP: other pathology (e.g. gliosis, cyst or atrophy). 
varying margins, including the amygdalo-hippocampal complex, and resection of lesions located in the neocortex when present. Postoperatively, no patients showed clinical decline in cognitive language and memory functioning. Resection volumes were derived from MR images. The right temporal lobe resection volume was on average 50\% larger $(p=0.01)$ than in the left temporal lobe due to the lack of clear restrictions to spare eloquent cortex. The medical ethical committee of the Maastricht University Hospital approved the protocol of the project, to which all subjects gave written informed consent.

\subsection{Language paradigms}

To test two language tasks were used: covert word generation and covert text reading. During word generation, the subjects had to covertly generate as many words as possible beginning with a presented letter (U, $\mathrm{N}, \mathrm{K}, \mathrm{A}, \mathrm{E}$, and P). The word generation task was conducted in a block-design time course in which one letter was presented per epoch and the baseline task comprised the viewing of a fixation cross. The reading task was performed in the same sequence in which the reading of a meaningful text was contrasted with reading of text containing pronounceable nonsense words. Each language block took $32 \mathrm{~s}$ and was conducted six times. Visual stimuli were projected with a beamer on a transparency screen and synchronised with the MRI system using custom-made software. The paradigm was previously tested in healthy volunteers (Deblaere et al., 2002; Aldenkamp et al., 2003). After the fMRI sessions subjects were asked to repeat as many as possible generated words and to tell the contents of the text. All subjects performed both language tasks sufficiently well.

\subsection{MR protocol}

Images were acquired on a $1.5 \mathrm{~T}$ MRI system equipped with a standard receiver head coil. For fMRI a single-shot multi-slice echo-planar $\mathrm{T} 2{ }^{*}$-weighted pulse sequence was applied covering the entire cerebrum. Imaging parameters were TR $4 \mathrm{~s}$, TE $50 \mathrm{~ms}$, flip angle $90^{\circ}$, matrix $64 \times 64$, pixel size $3.5 \mathrm{~mm} \times 3.5 \mathrm{~mm}$, slice thickness $3.5 \mathrm{~mm}, 34$ contiguous axial slices per volume, and 96 volumes per paradigm. For anatomical reference and to determine the resection volume, a
3D T1-weighted fast-field echo pulse sequence was conducted, with parameters TR $11 \mathrm{~ms}$, TE $3.5 \mathrm{~ms}$, flip angle $11^{\circ}$, matrix $256 \times 256 \times 150$, and voxel size $1 \mathrm{~mm} \times 1 \mathrm{~mm} \times 1 \mathrm{~mm}$. The images of the controls have been acquired in similar way as described elsewhere (Deblaere et al., 2002), but were analysed differently to be consistent with the patient data.

\subsection{Data analysis}

\subsubsection{Pre-processing}

Image processing and statistical analysis were performed using the software package SPM99 of the Wellcome Department of Cognitive Neurology (London, UK). Data pre-processing amounted to 3D motion correction, spatial normalisation to Montreal Neurological Institute (MNI) T2* image template, and spatial smoothing with an $8 \mathrm{~mm}$ Gaussian kernel. The anatomical images were also spatially co-registered to the corresponding MNI T1 image template. The images of the resected patients were spatially normalised to the template images by using affine transformations (i.e. rigid body, global scaling and skewing) only. Use of additional local deformations would erroneously stretch the cortex near the resected zone. Using affine transformations only, in the contrary to local deformations, preserved the shape of the resected volume in relation to the temporal lobe. Final voxel sizes were $2 \mathrm{~mm} \times 2 \mathrm{~mm} \times 2 \mathrm{~mm}$.

\subsubsection{Activation}

The amplitudes of the hemodynamic responses were obtained for each voxel in each subject by numerically fitting a model hemodynamic response function to the MRI signal time courses. Maps representing the significance of the brain activation amplitudes were obtained for each subject. Statistical inferences of individual voxels, in the regions of interest, were subjected to the problem of multiple comparisons of many brain voxels and the resulting $p$-values were corrected for the total number of spatially independent resolution elements (resels) (Holmes and Friston, 1997).

\subsubsection{Lateralization index}

The lateralization index (LI) was defined as the relative difference between the activation extent in specified regions of interest in the left and the right hemisphere. LI was computed for each subject using 
the formula $\mathrm{LI}=\left(V_{\mathrm{L}}-V_{\mathrm{R}}\right) /\left(V_{\mathrm{L}}+V_{\mathrm{R}}\right)$, where $V_{\mathrm{L}}$ and $V_{\mathrm{R}}$ represent the extent (i.e. number of voxels) of brain activation above the statistical threshold in the left and right hemispheres, respectively. LI values range between -1 and 1 , which represents complete right and left-hemispheric lateralization, respectively. Note that by using the LI, one corrects (i.e. normalizes) for inter-individual variations in the amount of brain activation. By displaying LI as a function of the threshold one moreover is able to index the level of activation. The choice of the optimal threshold value may be limited by upper and lower boundary statistical effects. The threshold value should on the one hand be high enough to exclude a relatively large number of voxels false-positively accepted for activation. On the other hand, the threshold value should not be too high in order to reduce the number of voxels false-negatively rejected for activation and to reduce the weight of the relatively high $\mathrm{T} 2{ }^{*}$ responses (i.e. high $Z$-values) of draining veins. To this end, for every individual the LI was calculated for a range of threshold values to find deviations from predefined threshold value. For statistical comparison between groups the threshold was set to $Z>3.8$ for the group averaged LI, which moreover corresponds to $p<0.05$ when corrected for multiple (voxel) comparisons.

\subsubsection{Regions of interest}

Rather than using voxel-by-voxel comparisons (as automated, e.g. in SPM) we decided to employ region of interest analysis. The rationale for this choice is that for many voxels no brain tissue is present in postoperative patients. Performing a voxel-by-voxel analysis would therefore not make much sense for voxels in the temporal lobe as the resected brain regions vary in size and shape between patients. Quantitative analysis, using digital brain masks (Maldjian et al., 2003) (Fig. 1), was restricted to regions of interest

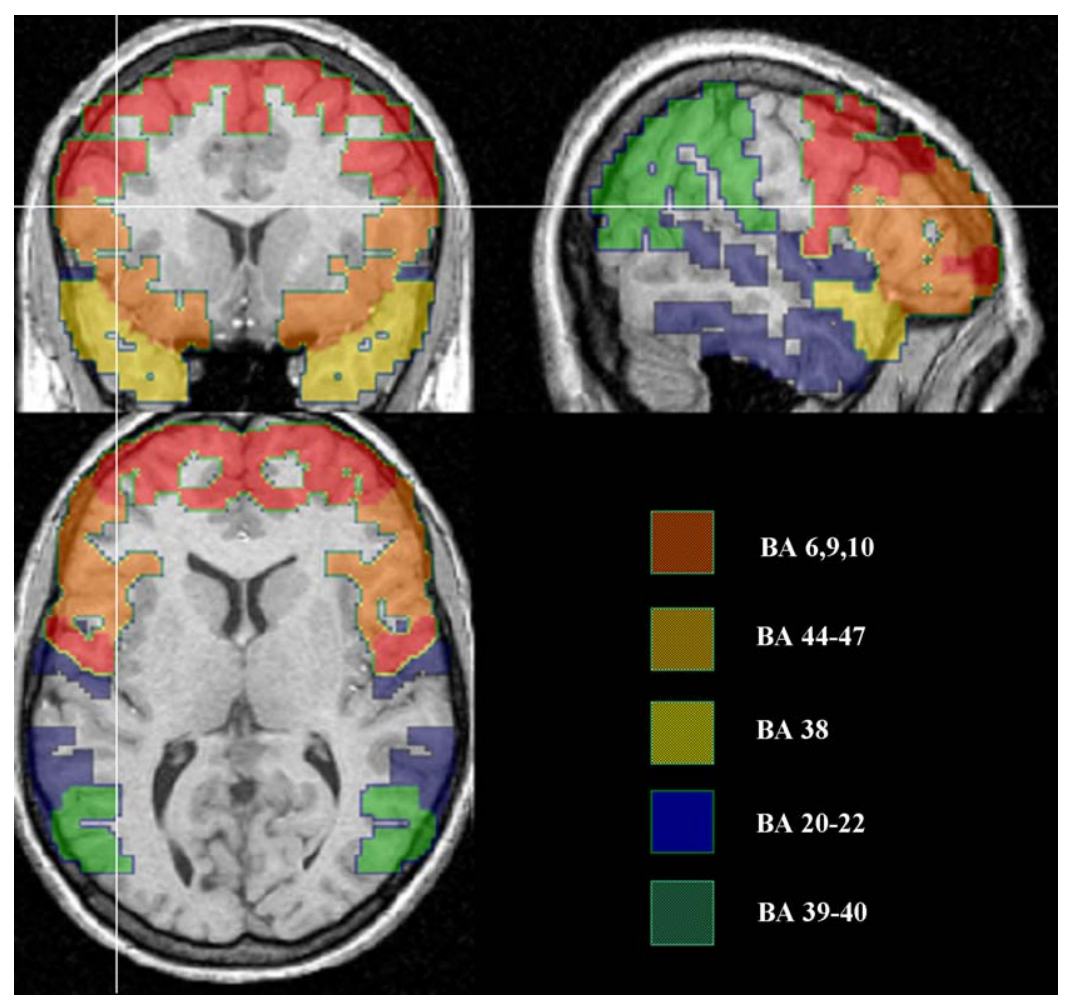

Fig. 1. T1 weighted images of a volunteer with color-coded brain mask comprising the inferior and middle frontal cortex (orange), the superior frontal cortex (red), the anterior temporal lobe (yellow), the posterior temporal lobe (blue), and the temporo-parietal junction (green). The color-coding of the pertaining Brodmann areas (BA) is depicted. 
that are known to be essential for language processing (Ojemann et al., 1989) and that have been previously identified to be activated in controls for the same language tasks (Deblaere et al., 2002). These regions comprise the inferior and middle frontal cortex (Brodmann areas, BA 44-47), the superior frontal cortex (BA 6, 9, and 10), the anterior temporal lobe (BA 38), the posterior and inferior temporal lobe (BA 20 and 21), and the temporo-parietal junction (BA 39 and 40). LI values of the language mask were classified to represent bilateral activation when $-0.2<\mathrm{LI}<0.2$.

\subsubsection{Distribution differences}

2.4.5.1. Lateralization. Relative changes of brain activation lateralization were quantified by calculating the differences of LI between RTL patients and controls, and between LTL and controls using the expressions $\Delta \mathrm{LI}_{\mathrm{RTL}}=\mathrm{LI}_{\mathrm{RTL}}-\mathrm{LI}_{\mathrm{N}}$ and $\Delta \mathrm{LI}_{\mathrm{LTL}}=\mathrm{LI}_{\mathrm{LTL}}-\mathrm{LI}_{\mathrm{N}}$, respectively. Here the lateralization index for healthy controls, RTL patients, and LTL patients is denoted as $\mathrm{LI}_{N}, \mathrm{LI}_{\mathrm{RTL}}$, and $\mathrm{LI}_{\mathrm{LTL}}$, respectively. Dominance of the left hemisphere, as is expected for language tasks in controls, is characterized by a positive $\mathrm{LI}_{\mathrm{N}}$. A relative shift of activation from the epileptic to the non-epileptic hemisphere is associated with significantly positive $\Delta \mathrm{LI}_{\mathrm{RTL}}$ for RTL patients and a significantly negative $\Delta \mathrm{LI}_{\mathrm{LTL}}$ for LTL patients.

Temporo-frontal distribution. Possible temporofrontal distribution changes were investigated by comparing the difference in the percentage of activated voxels between the temporal (BA 20-22, 39, 40) and frontal lobe (BA 44-47, 6, 9, 10) for each hemisphere and each task.

\section{Results}

\subsection{Word generation}

The control group as well as the RTL and LTL groups show a strong positive LI value for the defined language regions (Table 2). Fig. 2a shows that the LTL group has a reduced LI value compared to the control group for a broad range of threshold values. The asymmetry in the distribution between the hemispheres is mainly mediated by the strong left lateralized activation in the prefrontal cortex (example in Fig. 4).

The largest drop (20\%) of activation percentage for the LTL patient group was in the left frontal cortex (near Broca's region, BA 44-47) (Fig. 2b). The distributed activation percentage increased (about $11 \%$ predominantly in the BA 6,9,10 and 20-22) in the right hemisphere in the LTL patient group. The number of patients, moreover, showing an atypical language lateralization was the highest in LTL patient group (example in Fig. 4). None of the correlations between LI and the demographic parameters gender, age at onset, age at imaging, seizure frequency, interval between surgery and imaging, and resection volume were significant.

A significantly positive $\mathrm{LI}_{\mathrm{N}}(p \ll 0.001)$ was found, which indicates clear left-hemispheric lateralization for controls. The $\Delta \mathrm{LI}_{\mathrm{LTL}}$ was significantly negative, which implies a redistribution towards the righthemispheric activation in the LTL group $(p<0.03)$. No significant increases in $\mathrm{LI}_{\mathrm{RTL}}$ were found. Also no temporo-frontal distribution changes were found (Table 3).

Table 2

Group averaged lateralization indices (threshold $p<0.05$ or $Z>3.8$, corrected) for controls, right and left temporal lobe resected epilepsy patients, and the number of patients classified as right $(\mathrm{R}, \mathrm{LI} \leq-0.2)$, left $(\mathrm{L}, \mathrm{LI} \geq 0.2)$ or bilateral $\mathrm{B}(-0.2<\mathrm{LI}<0.2)$ representation

\begin{tabular}{lllllll}
\hline Task & Quantity & $\mathrm{LI}_{\mathrm{N}}$ & $\mathrm{LI}_{\mathrm{RTL}}$ & $\mathrm{LI}_{\mathrm{LTL}}$ & $\Delta \mathrm{LI}_{\mathrm{RTL}}$ & $\Delta \mathrm{LI}_{\mathrm{LTL}}$ \\
\hline Word generation & LI & $0.66(0.09)^{* *}$ & $0.53(0.09)$ & $0.31(0.17)$ & $-0.13(0.16)$ & $-0.35(0.17)^{*}$ \\
& $\mathrm{~L}: \mathrm{B}: \mathrm{R}$ & $8: 1: 0$ & $9: 2: 1$ & $5: 2: 2$ & & \\
Reading & LI & $0.59(0.12)^{* *}$ & $0.78(0.08)$ & $0.09(0.27)$ & $0.19(0.21)$ & $-0.50(0.23)^{*}$ \\
& L:B:R & $8: 1: 0$ & $11: 1: 0$ & $3: 3: 3$ & & \\
\hline
\end{tabular}

Values are mean \pm 1 S.E.M. RTL: right temporal lobe; LTL: left temporal lobe; N: control; LI: lateralization index. Also the differences in LI with respect to controls are listed.

* Statistical significance level: $p<0.03$.

** Statistical significance level: $p \ll 0.001$. 


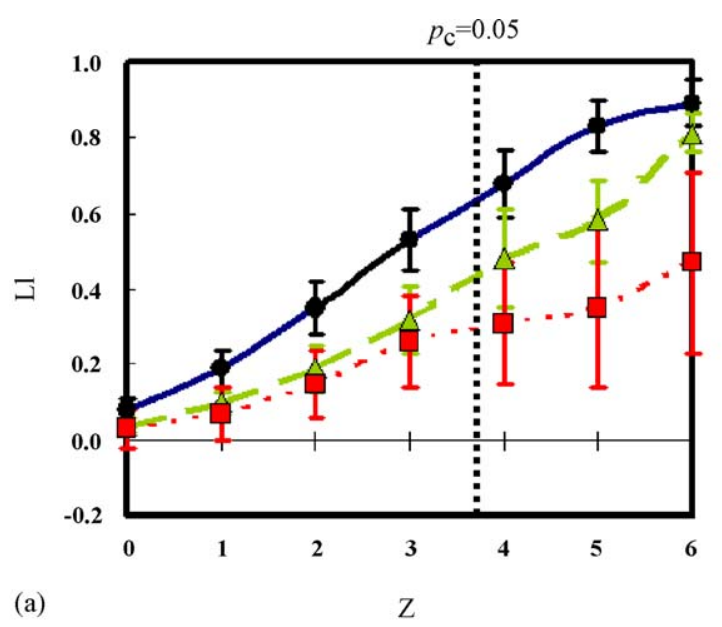

(a)

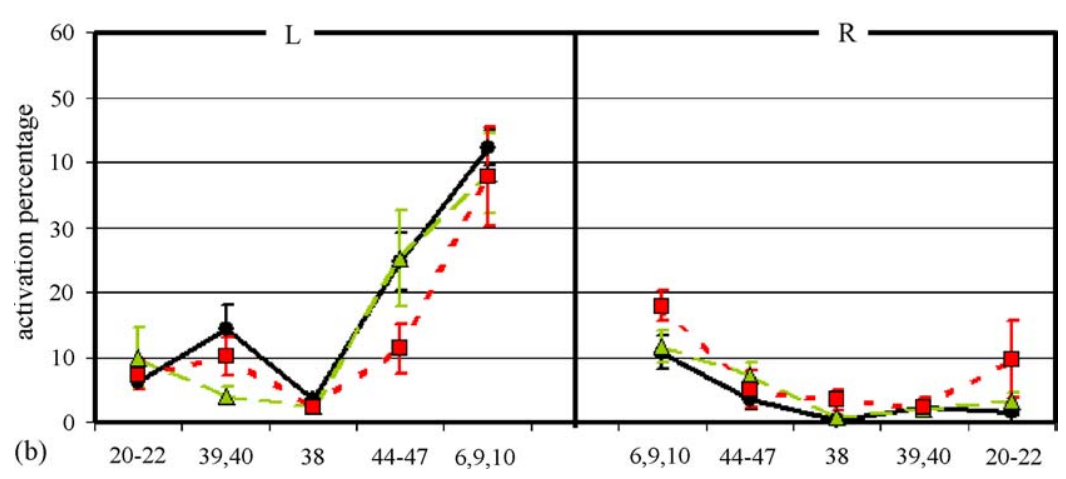

Fig. 2. The lateralization index (LI) as function of the statistical threshold value $Z$ for the controls (solid), the right temporal lobe (RTL) (dashed) and left temporal lobe (LTL) (dotted) patient group for the word generation task (a). The relative distribution of activated brain voxels (expressed in percentages) in each of the selected language-related regions (Brodmann areas) of the three groups (b). The error bars represent the standard error of the means.

Table 3

Group differences in percentage of activated voxels between the frontal and temporal lobe (threshold $p<0.05$ or $Z>3.8$, corrected) for controls, right and left temporal lobe resected epilepsy patients

\begin{tabular}{llccc}
\hline Task & Hemisphere & Controls & \multicolumn{1}{l}{ RTL } & LTL \\
\hline $\begin{array}{l}\text { Word } \\
\text { generation }\end{array}$ & $\mathrm{L}$ & $43 \pm 9$ & $47 \pm 12$ & $30 \pm 12$ \\
& $\mathrm{R}$ & $10 \pm 3$ & $13 \pm 3$ & $8 \pm 5$ \\
Reading & $\mathrm{L}$ & $-71 \pm 12$ & $-18 \pm 14^{*}$ & $-20 \pm 7^{* *}$ \\
& $\mathrm{R}$ & $-18 \pm 7$ & $4 \pm 3^{* *}$ & $-32 \pm 11$ \\
\hline
\end{tabular}

Values are mean \pm 1 S.E.M. RTL: right temporal lobe; LTL: left temporal lobe. Significant changes in temporo-frontal activation are indicated by one or more asterisks.

* Statistical significance level: $p<0.01$.

** Statistical significance level: $p<0.005$.

\subsection{Reading}

The control group shows a positive LI for the selected language regions (Fig. 3a and Table 2). The LTL group exhibit a LI $(Z>3.8)$ that is much lower than for the control and RTL group and approximately equal to zero for a broad range of thresholds, implying bilateral language representation. The LI curve of the RTL group lies higher than for controls. The spatial distribution among classical language areas shows an asymmetric pattern, which is mainly mediated by the strong left temporal lobe (BA 44-47 and 6, 9, 10) activation for the control and RTL group. The number of patients showing an atypical language lateralization was the highest in LTL patient group (example in Fig. 4). No significant relations were found between the 


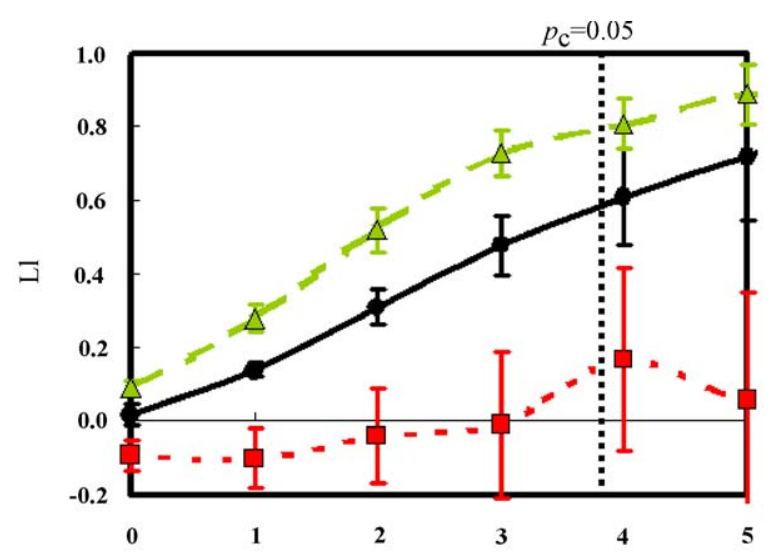

(a)

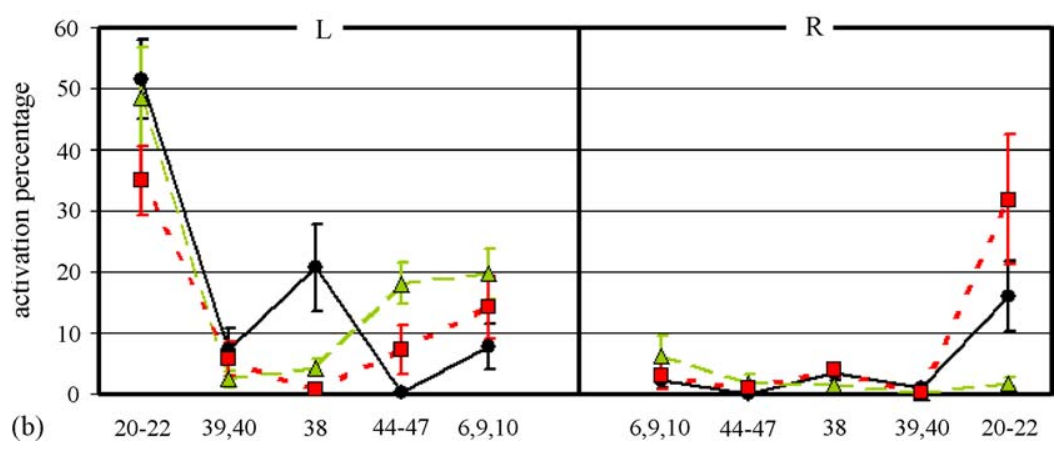

Fig. 3. The lateralization index as function of the statistical threshold value $Z$ for the controls (solid), the right temporal lobe (RTL) (dashed) and left temporal lobe (LTL) (dotted) patient group for the text reading task (a). The relative distribution of activated brain voxels (expressed in percentages) in each of the selected language-related regions (Brodmann areas) of the three groups (b). The error bars represent the standard error of the means.

LI and the demographic parameters age at onset, age at imaging, seizure frequency, interval between surgery and imaging, and resection volume.

For the LTL patient group the percentage of activation in the left posterior temporal lobe dropped by $16 \%$, while the right posterior temporal lobe increased by $16 \%$ (Fig. 3b). For the RTL patient group the left posterior temporal lobe activation remained unchanged, while the right posterior lobe decreased (about 14\%) and the left inferior frontal lobe increased (about 18\%) (Fig. 3b).

Temporo-frontal distribution changes were significant in both hemispheres in the RTL group and in the left hemisphere (only) of the LTL group (Table 3).

Again a significantly positive $\mathrm{LI}_{\mathrm{N}}(p \ll 0.001)$ was found, which indicates clear left-hemispheric lateralization for controls. The $\Delta \mathrm{LI}_{\mathrm{LTL}}$ was significantly neg- ative, which implies a redistribution towards the righthemispheric activation in the LTL group $(p<0.02)$. No significant increases in the $\mathrm{LI}_{\mathrm{RTL}}$ were found.

\section{Discussion}

Language activation assessed by fMRI, using word generation and reading tasks, was compared between patients with a right- or left-sided resection for temporal lobe epilepsy and with healthy controls. Although brain activation in response to language processing appeared in both hemispheres, a clear prevalent role of the left hemisphere was found only in controls and RTL patients. LTL patients, on the contrary, showed reduced left-hemispheric lateralization for word generation and showed bilateral activation for reading. Concordance 


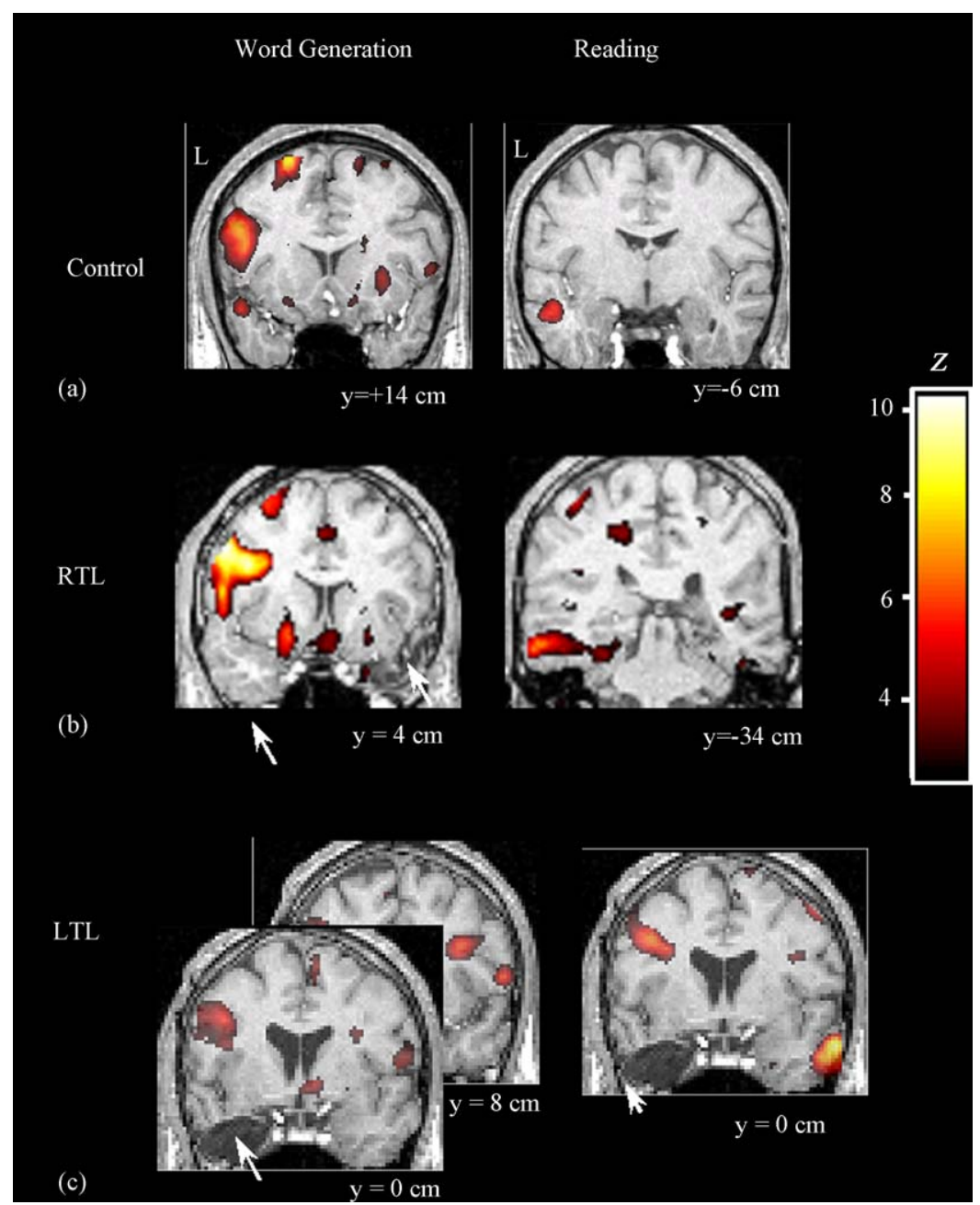

Fig. 4. The top row shows the (color-coded) activation map of a control subject for the word generation and the reading task superimposed on a T1 weighted image of one control. Clearly visible is the left lateralized prefrontal activation for word generation and the left lateralizaed temporal activation for the reading task. Also the homologuous areas in the contralateral hemisphere are activated but to a lesser degree (a). The middle row show the activation pattern of a patient of the right temporal lobe (RTL) group who yielded left lateralized activation for both tasks. The arrow points at the resected brain part (b). The lower row represents a patient of the left temporal lobe (LTL) group who showed bilateral activation for both tasks. The arrowhead points at the resected brain part (c). Note that the echo-planar T2*-weighted activation images had an original resolution of $3.5 \mathrm{~mm}$ and were co-registrated to the normalised T1-weighted 3D image with a final spatial resolution of $2 \mathrm{~mm}$. Possible geometric distortions on the echo-planar images due to field inhomogeneities may contribute to inaccurate co-registration.

with the pre-operative IAP language results is expectedly high for RTL patients but is surprisingly low for LTL patients. These results strongly suggests that an interhemispheric reorganisation of language activation has occurred in LTL patients.

This is the first imaging study demonstrating hemispheric differences in cerebral language activation between left- and right-resected patients. At first sight both the left-hemispheric epileptic seizures and the left temporal lobe resection might have contributed to the reduced left-hemispheric language lateralization in LTL patients. The data of this study do not allow to disentangle these two effects. Focal epileptic seizures underlying lesions with an onset early in life and the 
type of epileptic activity in the left hemisphere have already been reported to be the cause that a high percentage of LTL patients shows a bilateral language representation in the IAP (Helmstaedter et al., 1997; Risse et al., 1997; Janszky et al., 2003). This demonstrates that a slowly occurring reorganisation process may affect language organisation in patients with chronic refractory epilepsy. In the current study all patients, however, have been carefully selected to exhibit complete left-hemispheric language dominance according to the IAP that was assessed about 6 months before surgery. The possibility of (extensive) pre-operative language reorganisation is therefore unlikely. Moreover, only a limited number of patients (one LTL and one RTL) had a seizure onset before the age of 5, being the critical age to allow early language reorganisation. Adcock et al. (2003) have recently demonstrated that the LI values for preoperative LTL patients are lower than the RTL patients and controls. The preoperative LTL patient group, however, included 4 out of 12 patients with an atypical (one right and three bilateral) language lateralization according to the IAP in the contrary to the RTL group which included only patients with a left lateralized IAP result. Reanalysing the study by Adcock et al. (2003) shows that there are no differences in terms of LI values between pre-operative LTL and RTL patients when only patients with a lefthemispheric language dominance according to the IAP were selected. Because of the pre-operative language lateralization criterion and the almost perfect agreement between fMRI and IAP language lateralization reported in many studies (Binder et al., 1996; Yetkin et al., 1998; Lehéricy et al., 2000; Rutten et al., 2002a,b; Spreer et al., 2002; Adcock et al., 2003; Woermann et al., 2003) for RTL and LTL patients, the interhemispheric redistributions effects found in this study are more likely to occur due to the surgical intervention. Post-operative cerebral changes would moreover explain the rather large number of patients exhibiting disagreement between IAP and post-operative fMRI results as found in this study. This means that even in patients after left ATL, who show no obvious cognitive decline, the existing brain language system of the left hemisphere is affected and reorganized, despite all pre-operative precautions.

No significant effects of temporo-frontal distribution changes were observed for the word generation task in neither of the patient groups. This is most likely due to the fact that the activation for this expressive language task is mainly in the (left) prefrontal lobe. Significant temporo-frontal changes were however found for the reading task, which may be expected as this task evokes to a large extent the temporal lobe. For RTL patients the activation was decreased in both temporal lobes in comparison to the controls; this was compensated by increased (predominantly) left prefrontal lobe activation. For the LTL patients the activation in the right temporal lobe increased without strong right frontal activation. Left-hemispheric temporo-frontal changes occurred in LTL patients. These analyses reveal that damage (i.e. resection) to one cerebral node in the temporal lobe apparently affects the activation in the (remote left) frontal lobe.

One potential confounding mechanism that may have contributed to the bilateral balancing of activation in LTL patients is the volume loss of possibly activating tissue in the left ATL. This would suggest that our quantitative results represent a computational artefact. When the activation extent of the left ATL, however, is omitted in the calculation of the LI for the controls the LI turns out to be lowered by approximately 0.01 for the word generation task and by 0.1 for the reading task. This means that the resection of activated tissue does not fully account for the relative large drop in LI value for the LTL group. Moreover, volume loss would also not explain the increase in activation of regions in the contralateral hemisphere, which cerebral reorganization indeed does.

Standard ATL procedures, as performed in the patients included in this study, are usually smaller in spatial extent in the language dominant lobe than in the non-dominant lobe. This is reflected by the resection volumes which are on average 50\% smaller in the LTL than in the RTL patient group. Despite the smaller resection volume in the left temporal lobe, the strongest deviations in hemispheric activation patterns between controls and patients occur in the LTL patient group. This notion fits to the idea that during left ATL proper language areas or connections to them might be damaged, but remain unaffected during right ATL.

Interhemispheric reorganisations that occur during the time course of lesion development and epileptic seizure accumulation may evaluate gradually in time, and at the time of surgery may not be at the level to be detectable with the IAP. All patients exhibiting atypical language representation according the fMRI 
outcome in our study, do show, to some extent, remnant left-hemispheric brain activity. It remains unclear to which extent each activation site, whether left- or right-sided, contributes to the language processing. Moreover, the age at surgery for these patients was 18 years or higher, which is considered suboptimal for complete shifts of language function across the hemispheres. This suggests therefore, that in addition to the use of homologuous brain regions in the contralateral hemisphere preservation of the neural networks in the resected hemisphere exists. This mechanism has also previously been suggested for patients with language function recovery after stroke (Calvert et al., 2000) and patients with lesions with late onset (Müller et al., 1999). For the patients with left-sided resection in this study, however, it remains unknown whether the deviating activation patterns are related to recovery after impairment or not.

The fMRI technique is more sensitive to detect language capabilities of the right hemisphere than the IAP. Therefore, a comparison between pre-operative IAP results and post-operative fMRI results is complicated. Forthcoming studies intended to elucidate plasticity effects should feature pre-surgical imaging results as well. Such a study approach would also enable to demonstrate to which extent the right hemisphere is pre-operatively involved in language processing and avoid complications concerning the comparison between different techniques. Additional pre-operative fMRI would enable to directly measure activation changes yielding explicit evidence of acute redistributions effects in individual patients. Moreover, because LTL patients are much more at risk to exhibit post-operative declines in verbal memory and language functioning it would be interesting to investigate a possible relationship between cognitive declines as measured with neuropsychological tests and changes in brain activation distributions. A limitation of the current study is the clinical approach, thereby the absence of post-operative neuropsychological test results. Although no deficits were noted concerning language performance after surgery, subtle deficits may be detectable by detailed neuropsychological testing before and after surgery. Future work is encouraged to study TLE patients before and on several time-points after surgery. Ideally one would like to increase the number of patients included in such a longitudinal approach to track correlations between the changes in language organization, changes in neuropsychological performance, and patient characteristics as gender, onset of seizures, and seizure frequency, but also seizure spread and spiking frequency (Janszky et al., 2003). Moreover, it is noted that we used an observational study design that does not allow to conclude on causal relations instead of an experimental design.

In conclusion, hemispheric distributions of language activation obtained from post-operative fMRI exams highly differ between left and right ATL patient groups. Language activation patterns in patients after left ATL are less lateralized to the left hemisphere or are more bi-hemispherically distributed compared to both controls and RTL patients. Post-operative epilepsy patients also may have a significantly different temporo-frontal distribution of activation compared to controls. The results of this study on postoperative patients, in combination with recently published results on pre-operative patients (Adcock et al., 2003), suggest that resection of the left anterior temporal lobe results in interhemispheric redistribution effects for cerebral language organization, involving homologuous brain areas contralateral to the resected side.

\section{Acknowledgment}

This study is supported by grant number 99-02 of the National Epilepsy Foundation of The Netherlands.

\section{References}

Adcock, J.E., Wise, R.G., Oxbury, J.M., Oxbury, S.M., Matthews, P.M., 2003. Quantitative fMRI assessment of the differences in lateralization of language-related brain activation in patients with temporal lobe epilepsy. Neuroimage 18, 423-438.

Aldenkamp, A.P., Boon, P.A., Deblaere, K., et al., 2003. Usefulness of language and memory testing during intracarotid amobarbital testing: observations from a fMRI study. Acta Neurol. Scand. $108,147-152$.

Baxendale, S., 2002. The role of functional MRI in the presurgical investigation of temporal lobe epilepsy patients: a clinical perspective and review. J. Clin. Exp. Neuropsychol. 24, 664-676.

Bell, B.D., Davies, K.G., 1998. Anterior temporal lobectomy, hippocampal sclerosis, and memory: recent neuropsychological findings. Neuropsychol. Rev. 8, 25-41.

Binder, J.R., Swanson, S.J., Hammeke, T.A., et al., 1996. Determination of language dominance using functional MRI: a comparison with the Wada test. Neurology 46, 978-984. 
Calvert, G.A., Brammer, M.J., Morris, R.G., Williams, S.C., King, N., Matthews, P.M., 2000. Using fMRI to study recovery from acquired dysphasia. Brain Lang. 71, 391-399.

Clusmann, H., Schramm, J., Kral, T., et al., 2002. Prognostic factors and outcome after different types of resection for temporal lobe epilepsy. J. Neurosurg. 97, 1131-1141.

Davies, K.G., Bell, B.D., Bush, A.J., Hermann, B.P., Curtis Dohan, F., Jaap, A.S., 1998. Naming decline after left anterior temporal lobectomy correlates with pathological status of resected hippocampus. Epilepsia 39, 407-419.

Deblaere, K., Backes, W.H., Hofmann, P., et al., 2002. Developing a comprehensive presurgical functional MRI protocol for patients with intractable temporal lobe epilepsy: a pilot study. Neuroradiology 44, 667-673.

Helmstaedter, C., Kurthen, M., Linke, D.B., Elger, C.E., 1997. Patterns of language dominance in focal left and right hemisphere epilepsies: relation to MRI findings, EEG, sex, and age at onset of epilepsy. Brain Cogn. 33, 135-155.

Hermann, B.P., Wyler, A.R., Somes, G., Clement, L., 1994. Dysnomia after left anterior temporal lobectomy without functional mapping: frequency and correlates. Neurosurgery 35, 5257.

Hertz-Pannier, L., Chiron, C., Jambaqué, I., et al., 2002. Late plasticity for language in a child's non-dominant hemisphere. A preand post-surgery fMRI study. Brain 125, 361-372.

Holmes, A.P., Friston, K.J., 1997. Statistical Parametric Mapping, Chapter 3: Statistical Models and Experimental Design. Wellcome Department of Cognitive Neurology, http://www.fil. ion.ucl.ac.uk/spm/notes/course.html.

Janszky, J., Jokeit, H., Heinemann, D., Schulz, R., Woermann, F.G., Ebner, A., 2003. Epileptic activity influences the speech organization in medial temporal lobe epilepsy. Brain 126, 20432051.

Langfitt, J.T., Rausch, R., 1996. Word-finding deficits persist after left anterotemporal lobectomy. Arch Neurol. 53, 72-76.

Lee, T.M.C., Yip, J.T.H., Jones-Gotmannt, M., 2002. Memory deficits after resection from left or right anterior temporal lobe in humans: a meta-analytic review. Epilepsia 43, 283291

Lehéricy, S., Cohen, L., Bazin, B., et al., 2000. Functional MR evaluation of temporal and frontal language dominance compared with the Wada test. Neurology 54, 1625-1633.
Loring, D.W., Meador, K.J., Lee, G.P., et al., 1990. Cerebral language lateralization: evidence from intracarotid amobarbital testing. Neuropsychologia 28, 831-838.

Maldjian, J.A., Laurienti, P.J., Kraft, R.A., Burdette, J.H., 2003. An automated method for neuranatomic and cytoarchitectonic atlasbased interrogation of fMRI data sets. Neuroimage 9, 1233-1239.

Müller, R-A., Rothermel, R.D., Behen, M.E., Muzik, O., Chakraborty, P.K., Chugani, H.T., 1999. Language organization in patients with early and late left-hemisphere lesion: a PET study. Neuropsychologia 37, 545-557.

Ojemann, G., Ojemann, J., Lettich, E., Berger, M., 1989. Cortical language localization in left-dominant hemipshere. J. Neurosurg. 71, 316-326.

Rausch, R., Kraemer, S., Pietras, C.J., Le, M., Vickrey, B.G., Passaro, E.A., 2003. Early and late cognitive changes following temporal lobe surgery for epilepsy. Neurology 60, 951-959.

Risse, G.L., Gates, J.R., Fangmann, M.C., 1997. A consideration of bilateral language representation based on the intracarotid amobarbital procedure. Brain Cogn. 33, 118-132.

Rutten, G-J., Ramsey, N., Van Rijen, P., Alpherts, W., Van Veelen, C., 2002a. fMRI-determined language lateralization in patients with unilateral or mixed language dominance according to the Wada test. Neuroimage 17, 446-460.

Rutten, G.-J., Ramsey, N., Van Rijen, P., Van Veelen, 2002b. Reproducibility of fMRI-determined language lateralization in individual subjects. Brain Lang. 80, 421-437.

Spreer, J., Arnold, S., Quiske, A., et al., 2002. Determination of hemisphere dominance for language: comparison of frontal and temporal fMRI activation with intracarotid amytal testing. Neuroradiology 44, 467-474.

Springer, J.A., Binder, J.R., Hammeke, T.A., et al., 1999. Language dominance in normal and epilepsy subjects. A functional MRI study. Brain 122, 2033-2045.

Wada, J., Rasmussen, T., 1960. Intracarotid injection of sodium amytal for lateralization of cerebral speech dominance. J. Neurosurg. $17,266-282$.

Woermann, F.G., Jokeit, H., Luerding, R., et al., 2003. Language lateralization by Wada test and fMRI in 100 patients with epilepsy. Neurology 61, 699-701.

Yetkin, F.Z., Swanson, Fischer, M., et al., 1998. Functional MR of frontal lobe activation: comparison with the Wada language results. Am. J. Neuroradiol. 19, 1095-1098. 